Voix et Images

voixetimages

\title{
Écriture en locomobile
}

André Brochu

Volume 10, numéro 1, automne 1984

Littérature canadienne-anglaise

URI : https://id.erudit.org/iderudit/200465ar

DOI : https://doi.org/10.7202/200465ar

Aller au sommaire du numéro

Éditeur(s)

Université du Québec à Montréal

ISSN

0318-9201 (imprimé)

1705-933X (numérique)

Découvrir la revue

Citer cet article

Brochu, A. (1984). Écriture en locomobile. Voix et Images, 10(1), 151-162.

https://doi.org/10.7202/200465ar

Ce document est protégé par la loi sur le droit d'auteur. L'utilisation des services d'Érudit (y compris la reproduction) est assujettie à sa politique d'utilisation que vous pouvez consulter en ligne.

https://apropos.erudit.org/fr/usagers/politique-dutilisation/ 


\title{
Poésie
}

\section{Écriture en locomobile}

\author{
par André Brochu, Université de Montréál
}

Autres, autrement. L'hétérogène est le domaine d'élection de Michel van Schendel, dans son dernier recueil i. Hétérogène, d'abord, d'un discours poétique qui inclut son autre, le discours didactique, en plusieurs points du livre pour annoncer la couleur sémantique - une couleur éclatante, liée le plus souvent à la somptueuse désinence en -al: l'inaugural (p. 9), le banal (p. 18), le sensuel (p. 35), le monumental (p. 54), le proverbial (p. 65), l'hétérogène (p. 74), le mùsical (p. 93 ). Ces catégories sont autant de traits qui entrent dans la composition du texte. Banal, oui, car la circonstance quotidienne est le point d'envol fréquent du poème, 'qui pourrait toujours porter un titre semblable à celui-ci : «Écrit rue:Dantón près des yeux fertiles / ou Hommage à un livre»". Comment interpréter l'allusion, déniée par l'absence d'italique, au recueil d'Eluard? Est-il bien le livre auquel on rend hommage? Ou les «yeux»»ne sont-ils pas une présence aimée? Â vrai dire, les quelques vers du poème ne répondent guère à ces questions: Mais ce qui m'importe ici, c'est l'intrication extrême du littéraire et'du.vécu; laquelle réalise le titre d'Eluard en en faisant l'élément non signalé d'un autre titre; et c'est l'inscription dans l'intitulé lui-même de la: circonstance de lieu de l'écrire.

1. Michel van Schendel, Autres, autrement; Montréal, l'Hexagonè, 1983, 100 p. 
Le banal, chez van Schendel, reste rarement banal au-delà d'un vers ou deux. Sans qu'on délaisse le champ du concret, les mots sont vite retournés du côté où ils font ombre et déroutent le sens. On parlerait facilement d'hermétisme, ou encore de cérébralité. Là-dessus, le poète s'insurge :

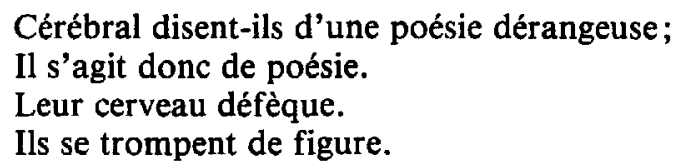

La vérité, c'est que van Schendel, à l'instar des vrais poètes, croit que la pensée peut favoriser la multiplication illimitée des résonances signifiantes. À partir, donc, du vécu spontané mis en vers, il organise de ces hyperprismes verbaux qui sont aussi des événements d'idées et des interventions politiques, au sens exact du terme. Le lecteur léger croit qu'il spécule, alors qu'il rend aux mots de tous les jours ce poids qu'ont seules les décisions de vie ou de mort. Langage capital, et non "de tête». Capital (encore la désinence en -al, accordée aux grandes choses), mais qui sait s'adoucir en tendres vocables: le cil, par exemple, où tremble l'intention du regard:

Je n'ai pas de plus grand souci

Que d'accroître d'un cil

L'extrême de ses pas

...

Elle n'a pas bougé d'un cil

L'oil est déçu d'un cil.

...

La flamme de l'huile et l'ombre des cils sur la peur du soleil.

$\ldots$

Elle allonge le cil qui s'éloigne vers les plantes de montagne.

On éprouve rarement l'impression, double, d'un recueil plus construit et d'une inspiration plus merveilleusement anarchique.

Les romantiques aimaient gravir une montagne et contempler, solitaires, le coucher du soleil - salutation de roi à roi sous l'aile de la mort. Jacques Brault aime aussi ces «moments fragiles » (c'est le titre de son der-

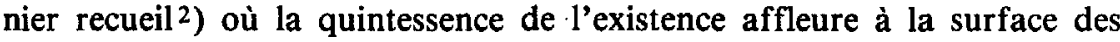
choses, faisant du cosmos le miroir du cour :

2. Jacques Brault, Moments fragiles, Saint-Lambert, le Noroît 1984, 114 p. 
Je gravis une colline

et je m'assois solitaire

sous un ciel vide

à mes pieds s'endort

comme un chien ma tristesse

C'est, décapé de toute rhétorique, le sentiment de Chateaubriand ou de Lamartine en ce qu'il a de plausible encore en notre siècle. Plus de lyrisme ici, car le lyrisme est éloquence (il y en avait encore, et du meilleur, dans la "Suite fraternelle» qui a propulsé Jacques Brault au rang des principaux poètes québécois ${ }^{3}$ ). Il reste, simplement, la suggestion. Les brefs poèmes qui forment les suites intitulées "Murmures en. novembre", "Amitiés posthumes ", "Vertiges brefs», "Leçons de solitude» et "Presque silence» sont de paradoxales élégies miniatures, où un incurable désespoir s'exprime sans révolte, en chuchotements brefs, en images doucement inouïes:

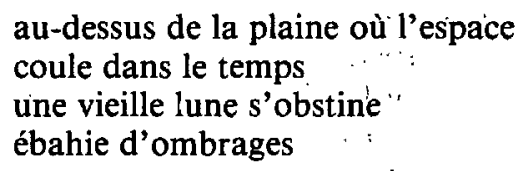

Que dit cette "vieille lune» ébahie : d'ombrages? L'obstination du poète, resté fidèle à lui-même, à une tristesșe que les modernes ont congédiée? Peut-être; mais on est rejoint surtout par la fraîche violence des contrastes: le lumineux et l'obscur, la véțusté et la soudạineté ( "ébahiẹ)). Les mots recréent tout un paysage, chargé de dynamisme, sur lequel on peut longtemps rêver car il réveille nos plus fervents souvenirs de ciel nocturne. De la même façon, ces deux vers parfaiț disent toutes nos forêts:

Est-ce une odeur de sapin que le profond de la forêt murmure

Une quasi-tautologie, que le rythme, essentiel et inanalysable, métamorphose en Réel (absolu). La poésié de Brault est une dés rares qui, audelà du langage, rend sensible l'existence dủ mondë.'

Alors que le recueil de van Schendeli, bien que fortement construit, accueillait la saveur hétéroclite des circonstances quotidiennes, le Livre du devoir, de Normand de Bellefeuille ${ }^{4}$, fait régner la rigueur jusque dans l'extrême détail du texte. Quatre fois quinze morceaux, généralement d'une page chacun; ces groupements portent le : nom d'actes, :comme dans une tragédie. Et c'est une tragédie, sans doute, que le poète y représente (ou irreprésente, car la lisibilité est d'emblée récusée, pas forcément au profit du

3. Dans Mémoire, Montréal, Déom, 1965,82 p.; p. 43-55.

4. Normand de Bellefeuille, le Livre du devoir, Montréal, les Herbes rouges, 1983, $100 \mathrm{p}$. 
scriptible comme le voulait Barthes, mais d'un illisible qui se suffit à luimême). La mort y est partout présente, «la mort sans doute épatante et prochaine», liée aux figures d'une jeune femme dont «la jupe remonte découvrant les jambes jusqu'à mi-cuisse" et d'une bête "entre les pieds qui sont droits», une bête «qui est droite et me regarde». Autour de ces éléments de représentation minimaux tournent quelques motifs incessamment repris, déformés, transformés, combinés de façon diverse : "le luxe maximum des animaux à écailles", "les chagrins de la matière", "véritable machine de détresse ", "les anneaux rutilants au bord de ta pensée», etc. Des bribes de discours rimbaldien s'y insèrent : «Ce ne peut être que la fin du monde, en avançant » ("Enfance», les Illuminations), «Et la Mère, fermant le livre du devoir...» («Les poètes de sept ans»). De ces formules, qui suggèrent beaucoup de sens et n'en fixent aucun, de Bellefeuille construit une fascinante machine verbale, 'qui donne au lecteur l'impression de progresser dans la compréhension d'un drame (bien saignant, comme il se doit), mais pour mieux le laisser en plan. Une citation de Derrida expose le programme: «Par endroits, je laisserai toute sorte de repères, des noms de personnes et des noms de lieux, des dates authentifiables, des événements identifiables, ils se précipiteront les yeux fermés, croyant enfin y être et nous y trouver quand d'un coup d'aiguillage je les enverrai ailleurs voir si nous y sommes, d'un coup de plùme ou de grattoir je ferai tout dérailler ... " Les repères sont la:figure d'un mort inconnu, Alain P., puis celles de la famille, grand-mère, mère, tante, oncle, grand-père paternel, grand-père maternel, grand frère, jeune soeur, tous unis et communiant par le sang en des rituels étranges ( « Je suis né aux cuisines, un soir de réveillon, tous ces sangs à table en mémoire, cette première mort comme prouesse et le repas de l'année neuve qui fait écho, figures réciproques du drame et de sa fable "). Mais parmi ces figures, il en est une qui fait voler la fable en éclats, empêche le drame de se ccaguler en événement: le père.

Le père échappe. Maintenant sans figure au bord de ma pensée. Père que dans la figure, le père échappe à la figure.

Il amène aussi le texte à sa conclusion, une conclusion ouverte, un recommencement. Étrange que celui qui représente la Loi et le devoir soit précisément, ici, celui qui les rend inopérants. Le père n'a pas - n'est pas - un nom mais un prénom:

Alors, patientant, réécrire les longues, trop longues syllabes du prénom de mon père, RAYER QUELQUES CODES.

Cela dit, on aurait tort de trop comprendre. 
Et, de comprendre, il n'est plus du tout question à la lecture de l'œuvre complète de Huguette Gaulin, rassemblée sous le titre magique de Lecture en vélocipède (qui a donné son nom à la célèbre collection) ${ }^{5}$. Comme l'écrit fort justement Normand de Bellefeuille dans sa préface, ici triomphe le mot : "Peu de véritables images, peu de procédés cotés «poétiques». Que le mot, matériau souverain, disséminé, posé çà et là, devant, derrière, placé sur la page». Le mot dans sa matérialité donc, son espace propre, entièrement libéré des contraintes sémantiques. Et non de la syntaxe, qui présente comme allant de soi les collocations proprement in-sensées. Les poèmes ont tous l'allure très sage de petits bouquets de vers libres. Qu'on y risque le nez cependant, et je ne sais quel parfum d'outre-Terre vous saisit:

spacieux portiques gantés d'arêtes
l'aquarium insère entre le suède du crâne
l'exasquelle
ses sociétés séductrices
que les déroulements de portes
mélancoliques n'interviennent
lors les enfances
doucement
déplacent les sucres

Comment dire avec plus de simplicité et d'élégance ce qui, à quelque point de vue qu'on se place, est à ce point dénué de pertinence que le pouvoir de suggestion des mots en est lui-même réduit à néant? Et pourtant, j'aime ces rencontres de mots: portiques gantés d'arêtes, sociétés séductrices; et il arrive même qu'une isotopie un peu consistante offre l'aumône d'un certain repos pour l'esprit, comme c'est le cas dans les derniers vers (enfance, douceur, sucre...). Mais comment m'assimiler le poème, qui de mot en mot me chasse de son centre (à vrai dire, il n'en a pas)? Il me faut alors lui imposer du sens, le mien - ou laisser couler. Par exemple, je puis décider que les «spacieux portiques gantés d'arêtes» suggèrent un passage à la fois offert et refusé (plus bas, le souhait que les «portes mélancoliques n'interviennent " prolongerait cette idée). Cette difficulté du passage est intérieure, concerne le "crâne » où l'imaginaire déploie ses mirages: j'interprète ainsi l' «aquarium» et ses "sociétés séductrices» par référence aux vers de «La fille maigre» d'Anne Hébert: «J'entrouvre / Mes prunelles liquides // Et bougent / Comme une eau verte / Des songes bizarres et enfantins ». L'exasquelle, mot forgé de toutes pièces, est une formation de l'imaginaire. Le passage effectué, on aboutit à l'enfance, tout comme dans le poème d'Anne Hébert. Et dans «Arbres» aussi, de Paul-Marie Lapointe.

Voilà donc une façon, certes non dénuée d'arbitraire, d'apprivoiser le texte et sans doute de le forcer, ou le tirer par les cheveux... Le lecteur, qui a

5. Huguette Gaulin, Lecture en vélocipède, Montréal, les Herbes rouges, 1983, $178 \mathrm{p}$. Préface de Normand de Bellefeuille. 
tous les droits, peut y recourir, histoire de s'assurer que le poème n'est pas un «bibelot d'inanité sonore » (Mallarmé). Autrement, qu'il se laisse griser par la vitesse des mots : le vélocipède est de qualité.

Aimer la poésie de Denis Vanier, c'est faire confiance au tigre qui, lui, n'a aucune raison de vous respecter. À tout moment, son souverain plaisir peut vous mettre en charpie. Ainsi, l'envoûtement que créent les mystérieuses formations verbales qui composent les poèmes de Rejet de prince $^{6}$ menace de céder la place à une violente déception. La charge d'arbitraire qui congédie la logique quotidienne n'est pas toujours compensée par la rigueur sous-jacente. Avec son auréole de poète maudit, de Rimbaud capable de toutes les outrances, Vanier écrit une poésie où la part de l'énonciation est particulièrement importante et indissociable de l'impression créée : on lit Vanier, à travers tel ou tel de ses textes, on subit l'arrogance de son hermétisme élégant, de ses images pas toujours heureuses ou éclairantes, on reçoit le coup au coeur de quelques vers étincelants de beauté (classique):

il coupe silencieusement l'arôme

de ses ongles roses

et quand elle pleure il pense au sérum

dans les cimetières vivants et le silence

La poésie-Vanier connote constamment les extrêmes: le délinquant, rôle dans lequel se complaît manifestement l'amateur de tatouages, l'auteur du poème intitulé «Police juvénile» (lequel a failli donner son titre au recueil, comme en témoigne une erreur dans l'impression de l'avant-titre); et le prince, c'est-à-dire le maître des mots et des rythmes, qui semble avoir un accès privilégié à l'âme du monde (c'est-à-dire, tout à la fois, de l'homme, des choses et du temps). Le thème des poèmes de Vanier, c'est toujours le verbe dont le mot âme est le sujet : aimer. Mais rien de l'amour à l'eau de rose; non plus que des grotesques convulsions sadiennes. Simplement l'intraitable désir d'un absolu :

désormais que des lacérations de tziganes
pour paroles
avec un grand vent bouilli
pour renverser nos châteaux étendus
dans la paix toujours prostrée
de la pure nécessité de l'absolu (...)

Le "grand vent bouilli», qui d'autre, dans la poésie actuelle, est capable de ça?

6. Denis Vanier, Rejet de prince, Montréal, VLB éditeur , 1983, 78 p. Préface de Suzanne Paradis, de l'Académie canadienne-française... 
Je me promets depuis longtemps de signaler le travail de Jean-Marc Desgent, qui n'est pas négligeable, même s'il m'inspire d'assez sérieuses réserves. L'occasion m'en est fournie par la publication de sa dernière plaquette, $O$ comme agression 7 . Certes, il s'agit de prose, bien que ce « récit». puisse être qualifié de poétique. À vrai dire, il oscille entre prose et poésie, d'une façon qui ne me semble pas particulièrement heureuse. On trouvait déjà, dans Faillite sauvage ou Transfigurations ${ }^{8}$, une certaine contamination du projet poétique par une intention prosaique, comme si le texte progressait à coups d'idées plus que par le déploiement de ses virtualités internes. Ici, la poursuite qu'on nous ráconte est sans cesse relancée de façon artificielle, sans qu'on en voie vraiment la nécessité. Lè narrateurprotagoniste, frère jumeau de celui de Prochain Épisode (une citation du roman précède d'ailleurs le texte), est pourchassé par "l'Órganisation" » qui est "peut-être le réel». Il a une acolyte, Suzie, qui est bien sûr absente et qu'il s'attend à retrouver à tout moment sur son chemin, comme l'énigmatique $\mathrm{K}$ d'Hubert Aquin. Les péripéties se succèdent sans que personnages et situations acquièrent la moindre consistance. Les fautes abondent: «la police tunésienne » (répété plusieurs fois), « pláttement»; le «cercle concentrique parfait" (comme si un cercle n'ettait pas concentrique par définition!), et des phrases comme: "Tous ceux qui désertent la mậchoire qui les désire».

Il me semble que, malgré un authentique désir d'écriré - un désir qui est un besoin -, Desgent n'a pas encore trouvé vraiment sa manière et 'ses thèmes, sa "personnalité littéraire». D'autres,'autour de lui, ont découvert la leur et l'exploitent sans vergogne. Desgent est loin de ce «carriérisme » et c'est heureux. Souhaitons seulement qu'il trouve un mode d'affirmation à la mesure de sa ferveur d'écrivain.

Avec un titre qui a le grand tort de rappeler un des plus purs joyaux du surréalisme, Travaux ralentis, de Pierre DesRuisseaux ${ }^{9}$, nous propose une poésie pleine de maturité et très personnelle; de celles qui ne font pas école et n'obéissent non plus à aucun magistère, parce qu'elles ont choisi de dire le fondamental. C'est une poésie qui, contrairement aux problématiques à la mode, célèbre la parole, la lumière et l'ouverture: «L'heure est venue comme si la lumière, pour celui qui désire la voir, ouvrait toute grande la terre ou la lune suppliante». Dans ce ton qui, parfois solennel, ne sombre jamais dans la grandiloquence, on retrouve la gravité sereine et sentencieuse

7. Jean-Marc Desgent, $O$ comme agression, Montréal, les Herbes rouges no 118, 1983, 36 p.

8. Jean-Marc Desgent, Faillite sauvage, Montréal, les Herbes rouges no 94 , août 1981, 40 p.; et Transfigurations, Montréal, les Herbes rouges no 106, octobre 1982, $36 \mathrm{p}$.

9. Pierre DesRuisseaux, Travaux ralentis, Montréal, l'Hexagone, 1983, 52 p. 
de la parole populaire, sur laquelle s'est penché l'auteur du Livre des proverbes québécois et du.Livre des expressions québécoises ${ }^{10}$. Mais rien de compassé. Des images d'une puissante beauté rendent tout à fait convaincant l'humanisme (et l'optimisme) du poète, telle cette «route... soulevée par l'extrême lumière d'une chose "; ou ces nuages «tachés de rues", dont l'invention témoigne d'une grande amitié pour le monde.

Tout n'est pas également inspiré dans ces poèmes brefs - ils ont rarement plus de six ou sept vers. On a parfois l'impression que le moulin à moudre du sens tourne à vide. Pourtant, même quand le poème tourne le dos aux fécondes évidences et semble s'enfoncer dans l'indécidable, il ne cesse de fournir matière à rêver. En voici un exemple, parmi beaucoup d'autres possibles:

Plus avant l'eau, soudain, se forme.

Oui, par nos mains

du fond de nos mains:

le reflet des télévisions

et le temps comme l'eau cousue approche.

On a l'impression, ici, d'une idée poétique intéressante mais insuffisamment mûrie, dont l'incomplétude se manifeste par les lacunes syntaxiques : reflet, sujet d'une proposition sans verbe, est coordonné à temps, sujet de approche. Ces entorses à la phrase n'empêchent pas de subir le choc de deux motifs, l'eau née des mains, qui affirme une continuité entre le corps et la nature; et le reflet (dans cette eau?) des télévisions, qui sont l'anti-nature par excellence: est-ce là subordonner la technique à la nature, aux éléments? Et subordonner, du même coup, le feu à l'eau? Il est intéressant de souligner une tendance à négliger ou refouler le feu, chez quelqu'un que son nom semble vouer au signe contraire; ainsi dans ce vers, où l'on reconnaît l'énumération des éléments, mais incomplète : «La terre précédée, l'air et l'eau.»

L'absence du feu, qui est énergie, expliquerait-elle les «travaux ralentis » du titre?

Avec le métier et la maturité acquis en quinze ans d'écriture, Madeleine Gagnon modifie sa manière habituelle et fait un retour au vers libre. Pensées du poème 11 est un recueil de textes brefs, lapidaires - à plus d'un

10. Pierre DesRuisseaux, le Livre des proverbes québécois, Montréal, l'Aurore, 1974; Hurtubise HMH, 1978. Le Livre des expressions québécoises, Montréal, Hurtubise HMH, 1979.

11. Madeleine Gagnon, Pensées du poème, Montréal, VLB éditeur, 1983, 64 p. 
titre puisque non seulement la phrase s'y fait volontiers sentence, mais l'inspiration y courtise ces épiphanies de la matière que sont les dioptases et chrysocolles (combinaisons du cuivre), saphirs, - chrysolithes; topazes, péridots, sardoines et j'en passe. Il ne s'agit pas d'extases, à la façon d'Anna de Noailles, devant les merveilles de ce monde mais d'un besoin de tabler sur la riche profusion de ce qui existe, pour mieux rejeter les mirages de l'idéalisme.

Or ce qui existe risque à tout moment de s'énoncer platement, surtoút quand on le met en rapport avec l'énigme de son apparition ou de sa fin. Le besoin, alors, de conjurer l'infini inspire au poète de laborieux clichés:

Le jour où je serai satiable
sera celui de ma mort
je serai rassasiée
quand je ne serai plus

De telles faiblesses font simplement ressortir les limites d'un parti pris métaphysique qui en vaut bien d'autres. On ne saurait éprouver que sympathie devant le tenace désir de s'installer au cœur même de l'espace réel: "Terre ouvre-toi / reçois-moi dans ton ventre / Astres accueillez-moi / au coeur de l'univers »; au coeur du temps réel aussi puisque le poète se perçoit comme le maillon d'une chaîne, fût-elle brisée:

Ce qui te précède
t'appartient
ce que tu laisses.
en partage
te quitte

Le début d'un autre poème réunit ces indications de position au centre du temps et de l'espace: "Sur l'électro-mondiogramme / porté en bandoulière au coeur / les bruits / du midi flamboyant ... " Le coeur du poète se confond avec le centre de l'univers et il est midi, l'heurre où ce qui est est (sacré saint Anselme!). L'heure où l'on assume pleinement son destin, sans le secours des dieux. Dieu, en vérité, on l'est soi-même: «J'écoute ma parole / parce que je suis dieu / Je suis tout pour moi / dans l'éternité / dans les siècles des siècles... (...)». Et tplus loin : «Ceci est mon corps / ceci est mon sang / ainsi je m'autorise / d'existence / ça n'est ni la fin du monde I ni le début des temps (...) ).

Et pourtant, même s'il est laïc et matérialiste, midi n'est pas l'Histoire puisque l'Histoire n'a pas de centre. Et elle ne se prend pas, que je sache, pour l'éternité! 
Le matérialisme de Renaud Longchamps, dans Miguasha ${ }^{12}$, n'a pas l'aspect un peu facile et sentimental qui caractérise celui de Madeleine Gagnon. À lire les distiques et tercets qui composent le recueil, on éprouve l'impression d'un langage très - construit, à la fois simple et opaque. L'hermétisme mécanique qui m'avait rebuté dans la majeure partie du recueil-rétrospective intitulé Anticorps ${ }^{13}$ me semble faire place ici à une autre sorte de discours, vastement illisible sans doute mais moins étranger à nos attentes de lecture, peut-être parce que le poème fait appel à un vocabulaire d'émblée chargé de sens:

\section{las de ses larves rouille sur mon rire (...) \\ je tiendrai par l'inertie à l'autre boue son sacrifice}

Le premier distique cité montre ce que la poésie de Longchamps doit aux allitérations (las-larves, rouille-rire) et autres jeux de sonorités (on lit ailleurs : «les lacunes de la lagune», «je sortirai de l'état avec l'éclat», et pis encore: «tous ces calculs du calcaire»!); le deuxième illustre les effets de sens suggérés à partir de mots fortement chargés de connotations. Le poème, chez Longchamps, est toujours le produit d'une négociation serrée entre une logique du signifiant et une logique du signifié. À l'intersection des deux se développe tout un réseau de mots récurrents, qui assurent une relative homogénéité de langage à ces fragments en liberté. Un des mots initiateurs, et ce n'est pas un hasard, est le mot père, qu'on retrouve en des contextes fort nombreux et différents; cela, on pourrait d'ailleurs le dire de tous les mots où se prolonge, en quelque sorte, le mot père : pierre, périr, perte, pèle (du verbe peler), peau, porte, partage, perpétuité, protéines, poitrail, pourrir. Ces mots reviennent avec trop d'insistance pour n'être pas les porteurs d'une intention, et leur matérialité les apparente, au sens actif du terme: «et ma perte que tu portes», «pourrir par la petite porte», «pour cent pères j'en viens à ma perte», "ce qui pourrit, heureusement, a péri», «je percuterai tous les pères du poitrail».

Une vague lueur de sens éclaire quelques-unes des «Quatre-vingts propositions de l'évolution». On y trouve ce constat enchanteur : «La vie comme maladie dè la matière», et cet autre, qui fait du sens une abstraction : «Sens de la vie hors de la vie». La matière exclut le sens : «Seul l'espace, la matière sur la matière. / S'appartenir et ne point signifier."

Une poésie matérialiste doit être lue les yeux fermés.

12. Renaud Longchamps, Miguasha, Montréal, VLB éditeur, 1983, $106 \mathrm{p}$.

13. Renaud Longchamps, Anticorps, Montréal, VLB éditeur, 1982, 378 p. 
Un premier regard sur Jours d'atelier, de Jean Royer ${ }^{14}$, nous projette dans l'espace multiple d'une bibliothèque et d'une galerie d'art. Pas un poème qui ne s'accompagne d'une épigraphe, ou encore qui ne se donne pour thème une citation ou l'œuvre d'un peintre ou d'un sculpteur (d'où le titre). Le danger des épigraphes, c'est qu'elles appellent la comparaison avec les vers auxquels elles introduisent, surtout quand leur présence tient du système. Mais leur raison d'être, on la trouve précisément dans une poésie que je qualifierai d'altruiste, une poésie qui est en son cœur même ouverture aux autres. Royer, tout en reconnaissant que le poème est affaire d'intimité, d'espace intérieur, et que «la poésie tient le seul langage qui va contre tous les autres langages ", trouve en lui-même une vocation spontanée au réel et à l'humain. Et l'humain, c'est d'abord la femme, l'amoureuse, objet d'une «intime soif» qui a son origine dans la "foetalité", où "l'enfant boit le songe de l'amour». Sans doute cette origine matricielle de l'amour explique-t-elle que l'être aimé, «l'alme», soit un chemin vers plus qu'elle-même : "Amoureux, toutes amours nous adorent ", et le désir fait boomerang, comme si l'autre se confondait avec la paroi maternelle du monde - un monde où tout est beau, tout est gentil, un monde du plain, pour reprendre un mot favori de Royer.

Sincère, généreuse, enthousiaste, cette poésie, comme toutes celles qu'anime un sentiment œcuménique, court le risque du convenu. Elle prétend y échapper, dans un groupe de poèmes intitulé «Noeuds», par l'usage de vieux mots rares, de préférence brefs: «moye», «hile», "ablais», «alumelle», "cueille», «noue», «navée», «nageret». Ils rendent les poèmes précieux et illisibles, mais jurent un peu avec le contexte.

Parfois, le journaliste se substitue carrément au poète pour écrire: "J'ajouterai avec Guy Viau, l'ami disparu, que Marcelle Ferron reste une force de la nature (...). Avec elle, la peinture est sœur de la poésie». De tels clichés n'ont rien à voir avec l'inspiration, et on mesure la distance entre ces "Ateliers» et les poèmes inventifs de Charron sur des motifs apparentés ${ }^{15}$.

Malgré ces faiblesses, Jours d'atelier nous fait entendre une voix d'une cordialité peu commune. Elle mérite bien que je la coiffe de mon point d'orgue quadrimestriel.

Non. Il me faut tout de même signaler deux revues de poésie, Lèvres urbaines qui, en janvier 1984, en était à sa sixième livraison, et Osiris, dont le numéro 17 est paru en 1983. Dans Lèvres urbaines, on lit des poèmes de Claude Beausoleil et Michael Delisle, les coordonnateurs, de Jean-Paul Daoust et de beaucoup de poètes confirmés, autrefois piliers de la Barre $d u$

14. Jean Royer, Jours d'atelier, Saint-Lambert, le Noroît, 1984, 94 p.

15. François Charron, D'où viennent les tableaux? Montréal, les Herbes rouges nos 110-112, $1983,96 \mathrm{p}$. 
jour et des Herbes rouges. Osiris, revue internationale publiée aux ÉtatsUnis, fait paraîtrẹ des poèmes en plusieurs langues, principalement l'anglais et le français. On y lit des textes de Gilles Cyr, Jean-Marc Fréchette, Charlotte Mélançon, Bernard Pozier, Joseph Bonenfant, Daniel Dargis, Pierre DesRuisseaux, Huguette Légaré, Jean-Pierre Issenhuth, Roland Giguère, Rina Lasnier, Robert Giroux, Robert Marteau et Carole Potvin. 